\title{
Influence of Supernova SN Ia Rate and the Early Star Formation Rate on the Galactic Chemical Evolution
}

\author{
Sandeep Sahijpal \\ Department of Physics, Panjab University, Chandigarh, India \\ Email: sandeep@pu.ac.in
}

Received July 12, 2013; revised August 13, 2013; accepted August 21, 2013

Copyright (C) 2013 Sandeep Sahijpal. This is an open access article distributed under the Creative Commons Attribution License, which permits unrestricted use, distribution, and reproduction in any medium, provided the original work is properly cited.

\begin{abstract}
Based on the recently developed numerical approach to understand the formation and the chemical evolution of the milky-way galaxy in the solar neighborhood we study the influence of the supernova type SN Ia rates on the galactic chemical evolution. Supernova SN Ia plays an important role in producing the iron inventory of the galaxy. We also study the dependence of the chemical evolution on the star formation rate prevailing during the initial one billion years of the evolution of the galaxy. This era marks the formation of the galactic halo and the thick disk. A comparison of the elemental abundance distributions of the dwarf stars in the solar neighborhood is made among the various models simulated in the present work. In order to explain the majority of the observed elemental evolutionary trends, specifically those related with the galactic evolution of iron and oxygen, it would be essential to incorporate a major component of prompt SN Ia to the galactic evolution. The prompt SN Ia would produce significant fraction of SN Ia within the initial $\sim 100$ million years from the time of star formation. The essential requirement of prompt SN Ia would result in a significant enhancement of SN Ia rates during the earliest epoch of the galaxy. The elemental evolutionary trends also favor an enhancement in the star formation rate during the initial one billion years of the galaxy at least by a factor of three compared to the trend prevailing during the latter evolutionary time of the galaxy.
\end{abstract}

Keywords: Galaxy Formation; Galactic Chemical Evolution; Stellar Nucleosynthesis; Star Formation; Supernovae

\section{Introduction}

The formation of the Milky-way galaxy commenced early after the big-bang around 13 billion years ago $[1,2]$. The primordial nucleosynthesis prior to the initiation of the formation of the galaxy led to the synthesis of the initial bulk inventories of hydrogen, helium and lithium. The heavy elements (including carbon and beyond), collectively referred as metals, were synthesized by successive generations of stars formed within the galaxy over the galactic timescales by a wide-range of nuclear processes. The galactic chemical evolution (GCE) deals with understanding the chemical evolution of the galaxy by following the evolution of the galaxy in terms of its gradual growth, the formation and evolution of successive generations of stars during the last 13 billion years (Gyr.) $[1,2]$.

The conventional approach adopted for the GCE is based on solving the set of classical integro-differential equations that deals with the evolution of the stable isotopes by taking into account the gradual accretion of the galaxy, the anticipated star formation rate and the stellar initial mass function at the time of formation of stars [1-6]. The stellar nucleosynthetic yields of the stars of distinct masses and metallicities are integrated with the equations to understand the isotopic evolution of the various elements over the galactic timescales. The conventional approach is based on the temporal evolution of the averaged behavior of the galaxy that in the case of the solar neighborhood is considered to be averaged over the spatial dimensions of the solar annular ring [e.g., 5]. The chemodynamical models of the GCE $[7,8]$ provide an alternative approach to understand the evolution of the galaxy in a more realistic manner as this approach incorporates the dynamical processes related with the evolution of the galaxy, the star formation and the mixing of the ejected stellar nucleosynthetic debris with the interstellar medium (ISM).

Another approach based on numerical simulations has been recently proposed to understand the GCE [9]. This direct approach is based on numerically simulating the entire evolution of the galaxy in terms of simulated episodes of successive generations of star formations in an 
evolving galaxy. The simulated stars evolve in a realistic manner according to their masses and metallicities, and eventually contribute their nucleosynthetic yields to ISM. The ISM gradually evolves in its elemental and isotopic composition. A wide-range of parameters related with the basic ingredients of GCE was studied to understand their dependence on GCE [9]. Apart from the latest suggestions regarding the star formation rate, the stellar initial mass function, the stellar nucleosynthetic prescripttions, the numerical approach [9] also incorporates the recent revisions in the solar metallicity [10]. The solar metallicity has been recently revised to a value of $\sim 0.014$ [10] from an earlier value of $\sim 0.02$ [11] on account of several modifications associated with the elemental abundance prediction of the solar photosphere. The reduction in the solar metallicity has resulted in a significant reduction in the anticipated supernova (SN II + Ib/c) rate history of the galaxy [9]. This is interestingly accompanied by almost negligible change in the star formation history of the galaxy [9].

Apart from understanding the origin of the bulk elemental and isotopic composition of the solar system around $\sim 4.56 \mathrm{Gyr}$. ago at the time of its formation [9], one of the main objectives of the GCE in the solar neighborhood is to explain the observed elemental abundance distribution of the F, G and K dwarf stars [12-14]. Due to the long life of the dwarf stars that is comparable to the galactic timescales, these stars serve as a proxy recorder of the elemental abundance evolution of the galaxy. While the spread in the observed elemental abundance distribution is generally associated either with the orbital diffusion of the stars over the galactic radial dimensions or the chemical heterogeneities in the GCE, the general trends in the temporal evolution of the various elemental abundances result from the gradual enrichment of the metallicity of the ISM on account of stellar nucleosynthetic contributions from several generations of stars $[1,2]$.

In the present work, we study the role of star formation rate in the initial one billion years of the evolution of the galaxy on the GCE. The initial one billion years correspond to the accretion of the halo and the thick disk of the galaxy [4]. This era corresponds to the formation of the metal-poor stars in the galaxy. Further, we study the dependence of the formulation adopted for the supernova $\mathrm{SN}$ Ia on the GCE. The supernova SN Ia is considered to be the main contributors of iron in the galaxy [3]. We present a representative set of simulations to understand the two major aspects studied in the present work. The preliminary results of this work were reported earlier in a conference [15].

\section{Supernova SN Ia Rate}

Due to the major contribution of iron to the galaxy [3], the SN Ia plays an important role in the chemical evolution of the galaxy specially during the initial stages of the formation of the galaxy. Despite of being a critical ingredient for the GCE, the SN Ia galactic rate is one of the most debatable issues $[6,7,9]$. There is no established standard formulation for predicting the SN Ia rate even though several suggestions have been made [e.g., 6,7,9].

Based on the conventional understanding, the binary stars in the mass range $3-8 \mathrm{M}_{\odot}$ and $11-16 \mathrm{M}_{\odot}$ are considered to eventually evolve to $\mathrm{SN}$ Ia. This could occur either in a single or a double degenerate binary system $[6,7]$. In the former scenario, the primary star within the binary system evolves to a white-dwarf before the secondary one evolves to the red-giant stage. Subsequently, the white-dwarf accretes matter from the redgiant and explodes as SN Ia. In this scenario, the lifetime of the secondary star that is less massive than the primary star determines the delay time of the SN Ia in the binary system since its formation. The shortest delay time within this scenario is based on the binary system with the total mass of $16 \mathrm{M}_{\odot}$. This corresponds to the secondary mass of $8 \mathrm{M}_{\odot}$ that has a lifetime of $\sim 40$ million years (Myr.). The longest delay time in this scenario would be of the order of the galactic timescales, the life-time of the secondary star in the low mass binary range of $3-5 \mathrm{M}_{\odot}$. Since the low mass binary systems would dominate the stellar initial mass distribution function for the star formation, the SN Ia rate distributions would be dominated by delay times of the order of billions of years in comparison with the prompt time delays of few $100 \mathrm{Myr}$. The double degenerate binary scenario involves merger, followed by SN Ia explosion, of C-O degenerate cores [6,7]. The progenitor masses of the two stars in this scenario are considered in the mass range of $5-8 \mathrm{M}_{\odot}$. The delay time in this scenario is determined by the mass of the secondary star in addition to the delay produced in the gravitational merger.

The observational records of SN Ia in various galaxies indicate a bimodal distribution in the delay time of SN Ia with a significant fraction of prompt SN Ia within $\sim 100$ Myr. with the remaining SN Ia exploding over a timescale up to $10 \mathrm{Gyr}$ [e.g., 6]. Based on the detailed analysis of the various proposed time delay distributions of SN Ia, the normalized time delay distribution corresponding to the single degenerate $\mathrm{SN}$ Ia scenario with a $\sim 13 \%$ contributions of the prompt SN Ia [6] provides the best description for the SN Ia formulation for GCE. We adopted this delay distribution in the present work to develop numerical simulations of the GCE. The results obtained from this model are compared with those obtained by using a model with a distinct procedure to formulate the SN Ia rate [9]. Further, we have also studied the role of the star formation rate during the initial one billion years on the chemical evolution of the galaxy. 
Based on the present work, we impose constraints on the star formation rate associated with the formation of the metal-poor stars in the solar neighborhood of the galaxy. All the simulations were performed to reproduce the revised solar metallicity of 0.014 [10] at the time of formation of the solar system around 4.56 billion years ago.

\section{Numerical Simulations}

The numerical simulations of the GCE were performed by evolving the isotopic composition of the stable isotopes from hydrogen to zinc in the ISM within the solar annular ring [9]. The width of the solar annular ring was assumed to be $\sim 1.9 \mathrm{kpc}$ [9], at a distance of $\sim 8 \mathrm{kpc}$ from the center of the galaxy. The galaxy was assumed to form in two episodes of exponential declining accretion rates [4]. The earliest era led to the accretion of the galactic halo and the thick disk over a timescale of one billion years, whereas, the accretion of the thin disk lasted over a timescale of 7 Gyr. The metallicity of the accreting matter was assumed to be 0.1 times the final acquired metallicity of the solar system in order to avoid the G-dwarf metallicity distribution problem [5,9].

The successive generations of stars were formed within the simulated ISM of the solar annular ring according to the star formation rate (SFR; in $\mathrm{M}_{\odot} \mathrm{pc}^{-2} \cdot \mathrm{Myr}^{-1}$ ) that depends upon the gas density $\left(\sigma_{\mathrm{Gas}}\right)$ and the total density $\left(\sigma_{\text {Total }}\right)$ of the ISM (Equation 1$)$ prevailing at any time $[5,9,16]$.

$$
\mathrm{SFR}=v\left[\left(\sigma_{\text {Total }}\right)^{0.33} \times\left(\sigma_{\text {Gas }}\right)^{1.67}\right] / \sigma_{\text {Total }}
$$

It should be noted that all the simulations were previously carried out with the value of unity for the parameter " $v$ " [9]. In the present work, the value of the constant multiplier, " $v$ " in the star formation rate was assumed to be unity for the accretion of the thin disk of the galaxy. However, in order to study the dependence of the GCE on the star formation rate in the earliest era of the galaxy formation that corresponds to the accretion of galactic halo and the thick disk, the value of the constant multiplier was varied. We ran three simulations with the values in the range of $1-3$ for the parameter " $v$ " (Table 1) to study the role of the era corresponding to the formation of the metal-poor stars on the evolution of elements in the galaxy. A higher value would correspond to an enhanced star formation rate during the initial $1 \mathrm{Gyr}$., and hence an enhanced early enrichment of the metallicity in the galaxy.

The simulations were performed with a time-step of 1 Myr. [9]. This timescale is around four times shorter than the life of the shortest lived star of mass $100 \mathrm{M}_{\odot}$ considered in the present work. Based on the star formation rate, an assessment was made after every time-step of $1 \mathrm{Myr}$. for the amount of ISM gas that will participate in star
Table 1. The distinct set of GCE models with the adopted simulation parameters.

\begin{tabular}{ccc}
\hline Model & $\begin{array}{c}\text { Star formation rate } \\
\text { parameter " } \boldsymbol{v} \text { " during } \\
\text { the initial 1 Gyr. }\end{array}$ & $\begin{array}{c}\text { The adopted supernova } \\
\text { SN Ia formulation }\end{array}$ \\
\hline Model-A & 1 & $\begin{array}{c}\text { Recently adopted } \\
\text { formulation [9] } \\
\text { (Prompt + delayed) } \\
\text { supernova SN Ia [6] }\end{array}$ \\
Model-B & 1 & $"$, \\
Model-C & 2 & $"$ \\
\hline
\end{tabular}

formation. On the basis of an assumed normalized stellar initial mass function, the stars of different masses, in the mass range $0.1-100 \mathrm{M}_{\odot}$, were formed in the simulations. We choose a three step normalized stellar initial mass function, $\varphi_{\mathrm{n}}(\mathrm{m}) \alpha \mathrm{m}^{-(1+\mathrm{x})}$, with three distinct power exponents " $x$ " [2,9] corresponding to the mass range of $0.1-1$ $\mathrm{M}_{\odot}, 1-8 \mathrm{M}_{\odot}$ and $11-100 \mathrm{M}_{\odot}$. The three power exponents were chosen to be $0,1.7$ and $\sim 1.5$, respectively for the three mass ranges. There is a slight variation in the third power index among the various simulations as this index is fine-tuned as one of simulation parameters to reproduce the solar metallicity at the time of formation of the solar system around 4.56 Gyr. Since, we simulate the evolution of the galaxy by simulating formation of stars, the mass range within $3-100 \mathrm{M}_{\odot}$ stars was covered by a discrete set of integer mass stars. However, in order to account for the abundant presence of the low mass stars in the galaxy, the low mass range was appropriately covered by the simulated stars with the assumed masses of $0.1,0.8,1.0,1.25,1.75,2.5 \mathrm{M}_{\odot}$ [9].

Based on the star formation rate and the assumed stellar initial mass function, the simulated stars were formed and evolved over their lifetimes according to their masses and the metallicites [1]. Subsequent to their evolution, the stars eject their nucleosynthetic products into ISM. Successive generations of stars were formed and evolved, thereby, enriching the ISM with nucleosynthetic products. The low and the intermediate mass stars with masses $\leq 8$ $\mathrm{M}_{\odot}$ evolve through AGB stage before becoming whitedwarfs. We incorporated the metallicity and mass dependent stellar nucleosynthetic yields of the AGB stars [17]. The single massive stars in the mass range $11-100$ $\mathrm{M}_{\odot}$ eventually explode as supernova $\mathrm{SN}$ II $+\mathrm{Ib} / \mathrm{c}$. We included the mass and metallicity dependent stellar yields of the massive stars [18]. Due to the uncertainties in the stellar physics of the stars in the mass range $8-11 \mathrm{M}_{\odot}$, the range was not included in the simulations. The nucleosynthetic yields of the massive stars beyond $25 \mathrm{M}_{\odot}$ [18] were obtained by averaging out the yields of the various stellar models with distinct remnant masses. The iron yields of $\mathrm{SN} \mathrm{II}+\mathrm{Ib} / \mathrm{c}$ of these massive stars were 
reduced by a factor of two as a standard practice [9]. The available stellar yields of different stellar masses and metallicities corresponding to the AGB stars and the massive stars were interpolated/extrapolated to obtain the stellar yields for the remaining stellar masses and metallicities required during the evolution of the galaxy. In the case of supernova SN Ia, we considered the metallicity independent yields of the various proposed SN Ia models [19]. Based on an equally probable random number generator, the various SN Ia models [19] were randomly triggered at the time of the supernova. This helps in averaging out the various uncertainties associated with the various $\mathrm{SN}$ Ia models.

As one of the main objectives of the present work is to understand the dependence of the GCE on the adopted supernova SN Ia formulation, we ran two set of simulations with two distinct formulations for the predicted supernova SN Ia rates (Table 1). These two formulations will determine the rate at which iron is synthesized in the galaxy, specifically during the initial stages of the evolution of the galaxy.

The SN Ia rate adopted in the Model-A (Table 1) is based on the formulation used in the recent GCE model [9]. According to this formulation, a fraction, " $\mathrm{f}$ " of the stars produced at the time of star formation according to the stellar initial mass function is treated as binary stars. These binary systems eventually evolve to SN Ia after a time delay that depends upon the lifetime, and hence, the mass of the secondary star in the binary system. As mentioned in the previous section, the single degenerate models of the SN Ia can evolve from the binary systems having the mass range of $3-16 \mathrm{M}_{\odot}$. The mass ratio of the secondary star to the primary star is derived observationally and indicates a ratio close to unity [2]. Based on this observation, the mass range of the secondary stars in the above mentioned binary systems was assumed to be in the range of $\sim 1.5-8 \mathrm{M}_{\odot}$ [9]. The secondary stars of these masses will evolve over the times scales that range from billions of years to $\sim 40 \mathrm{Myr}$. and trigger SN Ia of the primary star by shedding their matter to the latter during the red-giant stage. This formulation will result in a delayed SN Ia due to the nature of the stellar initial mass function that will produce lesser number of intermediate mass secondary stars in the mass range $3-8 \mathrm{M}_{\odot}$ compared to those in the range of $1.5-3 \mathrm{M}_{\odot}$. Even though this formulation will produce prompt SN Ia within a time-span of $\sim 100$ Myr., initiating within a minimum of 40 Myr., the fraction of such SN Ia will be low compared to those that are triggered over a comparatively longer timescale.

In the present work, we make comparison of the above adopted SN Ia formulation with the formulation based on the single degenerate SN Ia with the prompt SN Ia [6]. As mentioned in the previous section the latter formula- tion with a $\sim 13 \%$ contribution of prompt SN Ia apart from the delayed SN Ia over billions of years provides the best description for the GCE compared to the other formulations proposed earlier [6]. We adopted the normalized time delay distribution function corresponding to the single degenerate SN Ia model [6] to numerically simulate the GCE in case of the models B-C (Table 1). Since, the present approach of numerically simulating the GCE is based on considering the formation of successive generations of stars in a discrete manner compared to the other GCE models developed earlier that are based on the averaged evolutionary behavior of the galaxy [3-6], the normalized SN Ia time delay distribution function was discretized on the basis of SN Ia time delays into 25 temporal bins on the logarithmic scale initiating from 7.55 to 10 . The lowest one corresponds to the most prompt SN Ia occurring over a time delay of $\sim 40 \mathrm{Myr}$. This corresponds to the SN Ia from the binary system with the $8 \mathrm{M}_{\odot}$ secondary star. A fraction, "f" of the stars in the mass range of $3-16 \mathrm{M}_{\odot}$ at the time of a single episode of star formation was treated as the SN Ia progenitor binary systems. A single pool of these progenitor binary systems was created and triggered as SN Ia according to the normalized and binned time delay distribution function. Successive episodes of star formations will result in successive series of pools of the SN Ia progenitor binary systems.

It should be noted that the fraction, "f", of the stars generated as binary systems and the power index, " $x$ ", in the mass range 11-100 $\mathrm{M}_{\odot}$ of the stellar initial mass function are treated as the two simulations parameters in order to reproduce the solar metallicity at the time of formation of the solar system around 4.56 Gyr. Ago [9]. We obtained almost identical values, with slight variations, of $\sim 0.04$ and $\sim 1.5$ for "f" and " $\mathrm{x}$ ", respectively, in all the four simulations in the present work.

\section{Results and Discussion}

A representative set of four simulations were performed in the present work to understand the role of SN Ia rates and the early star formation rate on the galactic chemical evolution (Table 1). The deduced star formation rates for the four models are presented in Figure 1. The enhanced star formation rate in the case of Models C and D during the initial one billion years, the era corresponding to the formation of the galactic halo and the thick disk, results in a slightly lower star formation rate in the early era corresponding to the formation of the galactic thin disk due to the reduction in the available gas density following an earlier high star formation.

The deduced evolution of the total density and the gas density of the solar annular ring is presented in Figure 2. As mentioned above, the high early star formation rate in 


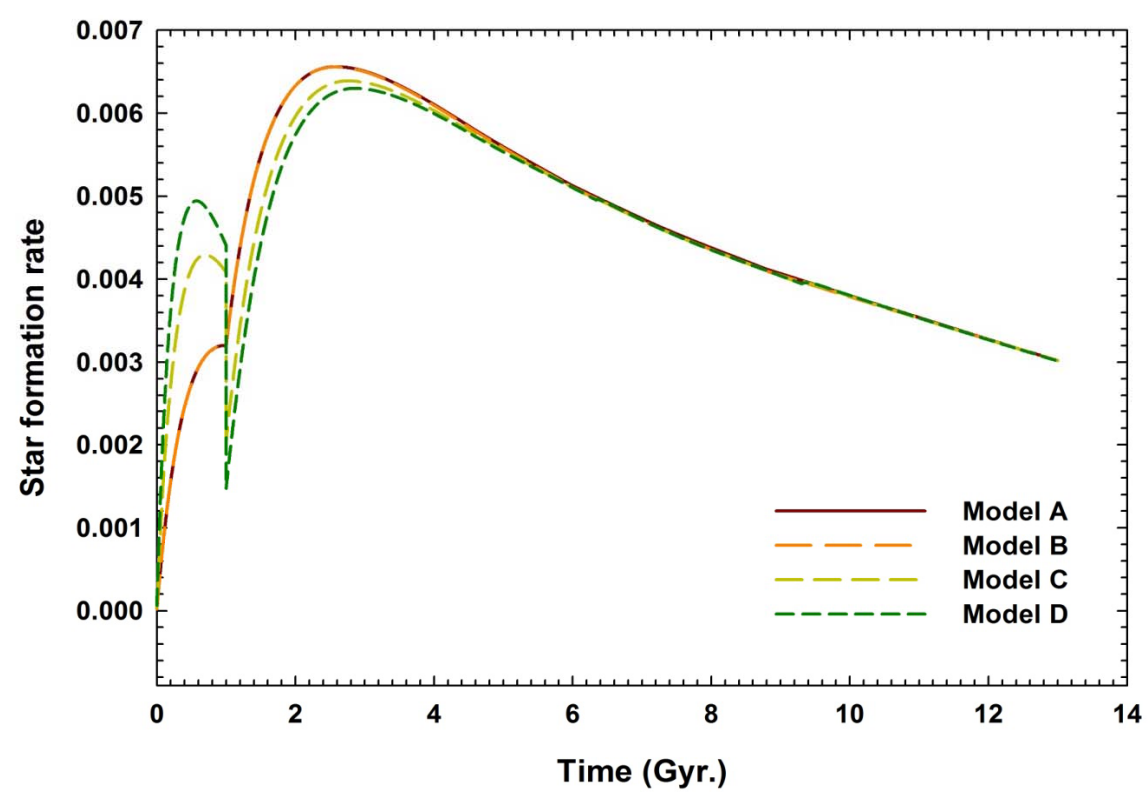

Figure 1. The deduced star formation rate $\left(\mathrm{M}_{\odot} \mathrm{pc}^{-2} \mathrm{Myr}^{-1}\right)$ for the various models in the solar annular ring.

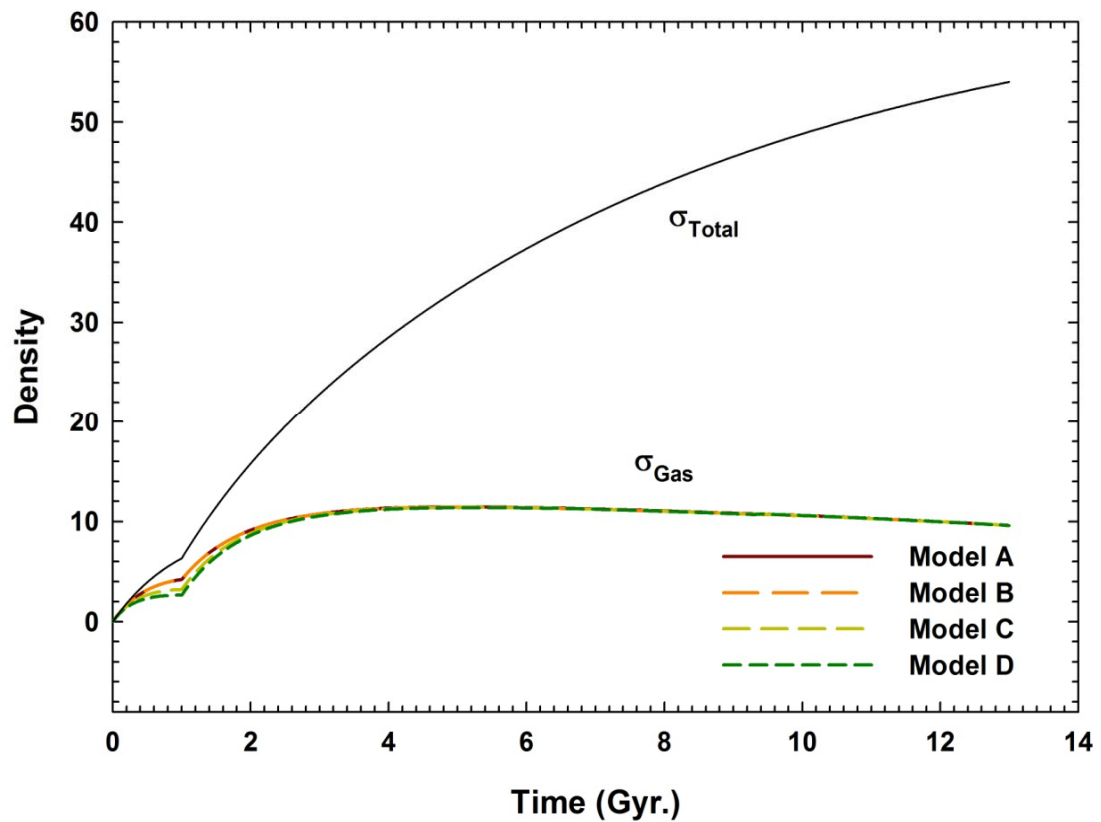

Figure 2. The deduced total surface density $\left(\mathrm{M}_{\odot} \mathrm{pc}^{-2}\right)$ and the gas density $\left(\mathrm{M}_{\odot} \mathrm{pc}^{-2}\right)$ for the evolution of the solar annular ring. The present predicted total surface density of $\sim 54 \mathrm{M}_{\odot} \mathrm{pc}^{-2}$ matches with the present observed value.

the models $\mathrm{C}$ and $\mathrm{D}$ results in a lower gas density in the subsequent time. This is marked clearly in the Figure 2. The deduced gas densities acquire identical values after 3 Gyr. Based on the star formation rates and the evolution of the gas density it could be deduced that the early enhancement of the star formation simply reschedules the star formation from the early galactic thin disk era to the thick disk era.

The growth of the metallicity in the solar annular ring on account of contributions from successive generations of star is presented in Figure 3. There is an insignificant change in the evolution of metallicity for the models A and $\mathrm{B}$ that corresponds to the two distinct formulations for the SN Ia. This is anticipated as the SN Ia is considered as the main source of iron whose abundance alone can be significantly altered by the distinct choice of SN Ia rates. The increase in the early star formation rate results in an increased early rise in the metallicity during the initial 1 Gyr. This is followed by a sharp fall on account of the declined star formation rate in the next 1 Gyr. 


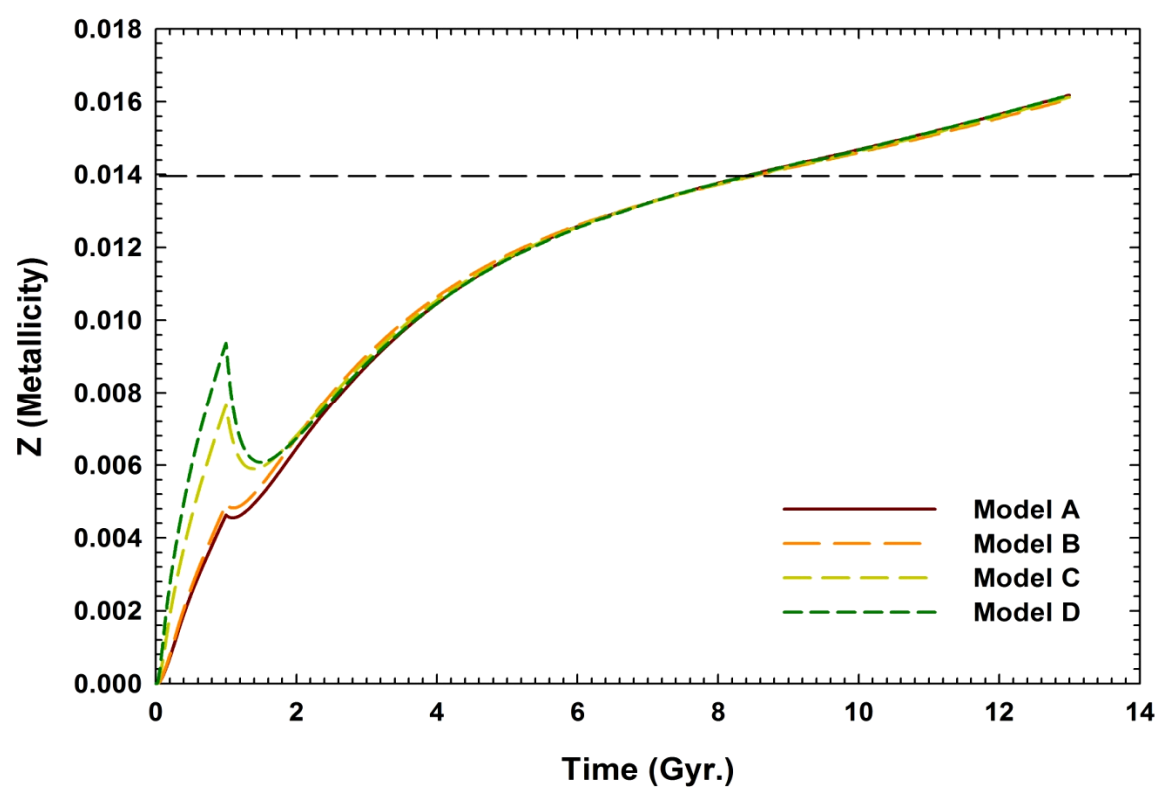

Figure 3. The deduced metallicity of the solar annular ring for the various models. The horizontal dashed line represents the solar metallicity of $\mathbf{0 . 0 1 4}$. All the models evolved through this metallicity at the time of formation of the solar system around 4.56 Gyr. ago.

The evolution of the metallicity beyond 3 Gyr. is almost identical in all the models. As mentioned earlier, it should be noted that all the simulations were performed to reproduce the revised solar metallicity of 0.014 [10] at the time of formation of the solar system around 4.56 billion years ago.

The deduced evolution of $[\mathrm{Fe} / \mathrm{H}]$ is presented in Figure $\mathbf{4}$ for the various models. There is a significant change in the early $[\mathrm{Fe} / \mathrm{H}]$ evolution in the case of the models A and B that correspond to two distinct SN Ia formulations. As SN Ia are the main source of iron in the galaxy, the model B with prompt SN Ia (Table 1) results in an early high $[\mathrm{Fe} / \mathrm{H}]$ values that reaches almost close to the $[\mathrm{Fe} / \mathrm{H}]$ observational values of the dwarf stars in the solar neighborhood. There is a further improvement in the early high production of iron in the case of Models C and D that correspond to an enhanced star formation rate in the initial 1 Gyr. of the evolution of the galaxy. The model D has the closest match with the observed data. The decline in the production of iron after the initial $1 \mathrm{Gyr}$. is due to the reduction in the star formation rate. The evolutionary trends of $[\mathrm{Fe} / \mathrm{H}]$ become almost identical for all the models after the initial $\sim 5$ Gyr. Beyond this time, the choice of distinct SN Ia formulations will probably have not much influence on the bulk GCE. Based on the $[\mathrm{Fe} / \mathrm{H}]$ evolutionary trend it can be summarized that the observed iron abundance distribution of the metal-poor dwarf stars in the solar neighborhood favors the SN Ia formulation with a prompt SN Ia and an enhanced star formation rate in the earliest epoch of the galaxy.
The deduced supernovae rates SN II + I b/c and SN Ia for the various models are presented in Figure 5. As the supernovae $\mathrm{SN} \mathrm{II}+\mathrm{Ib} / \mathrm{c}$ of the various massive stars that are associated with a single stellar cluster occurs within a timescale of $\sim 25 \mathrm{Myr}$. after the formation of the stars, the deduced trends in the $\mathrm{SN} \mathrm{II}+\mathrm{Ib} / \mathrm{c}$ for the various models correlates with the star formation trends (Figure 1). In the case of models B-D that are based on the SN Ia formulation that include prompt SN Ia (Table 1), the identical trend is also observed in SN Ia rates as the occurrence of SN Ia from the stars originating from a specific stellar cluster initiates around $\sim 40 \mathrm{Myr}$. after the formation of the stellar cluster and continues for more than billion years. These models result in an enhanced SN Ia contributions to the ISM within the initial 1 Gyr., the era that corresponds to the accretion of the galactic halo and the thick disk. This results in an enhanced production of iron as discussed earlier. On the contrary, the model A results in a delayed SN Ia by at least a time span of 1 $1.5 \mathrm{Gyr}$. as is evident from the time delays in the maxima of the SN Ia rate with respect to the maxima of the SN II $+\mathrm{I} \mathrm{b} / \mathrm{c}$ rates (Figure 5). The enhancement of the star formation rate in the early 1 Gyr. results in the further enhancement of the supernovae rates. The trends correlate with the star formation rates (Figure 1) and the gas density of the ISM (Figure 2).

The deduced normalized elemental abundance $[\mathrm{M} / \mathrm{Fe}]$ evolution of twelve elements with respect to $[\mathrm{Fe} / \mathrm{H}]$ is presented in Figure 6. Here, "M", represents one of the twelve elements ranging from $\mathrm{C}$ to $\mathrm{Zn}$. In order to make comparison of these predictions with the observations, 


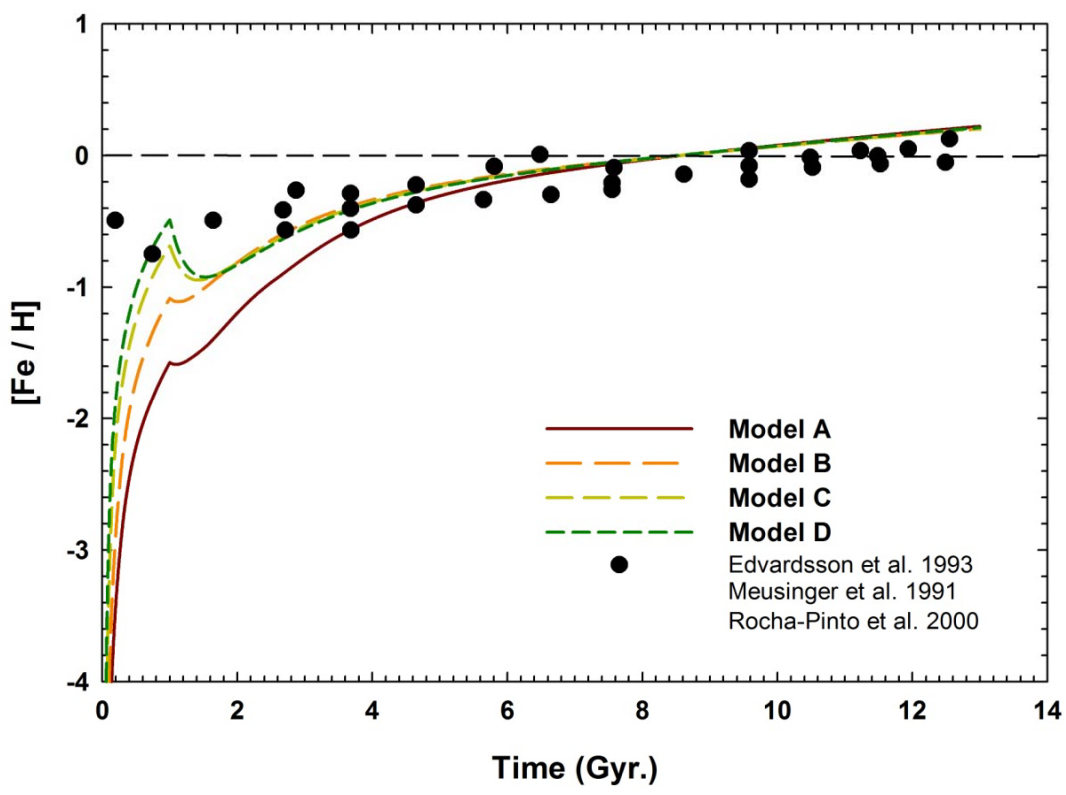

Figure 4. The deduced $[\mathrm{Fe} / \mathrm{H}]$ of the solar annular ring for the various models. The horizontal dashed line represents the reference solar value $[\mathrm{Fe} / \mathrm{H}]=\mathbf{0}$. All the models were evolved through this value at the time of formation of the solar system around 4.56 Gyr. ago. The observed data for the dwarf stars in the solar neighborhood is presented for comparison with the models.

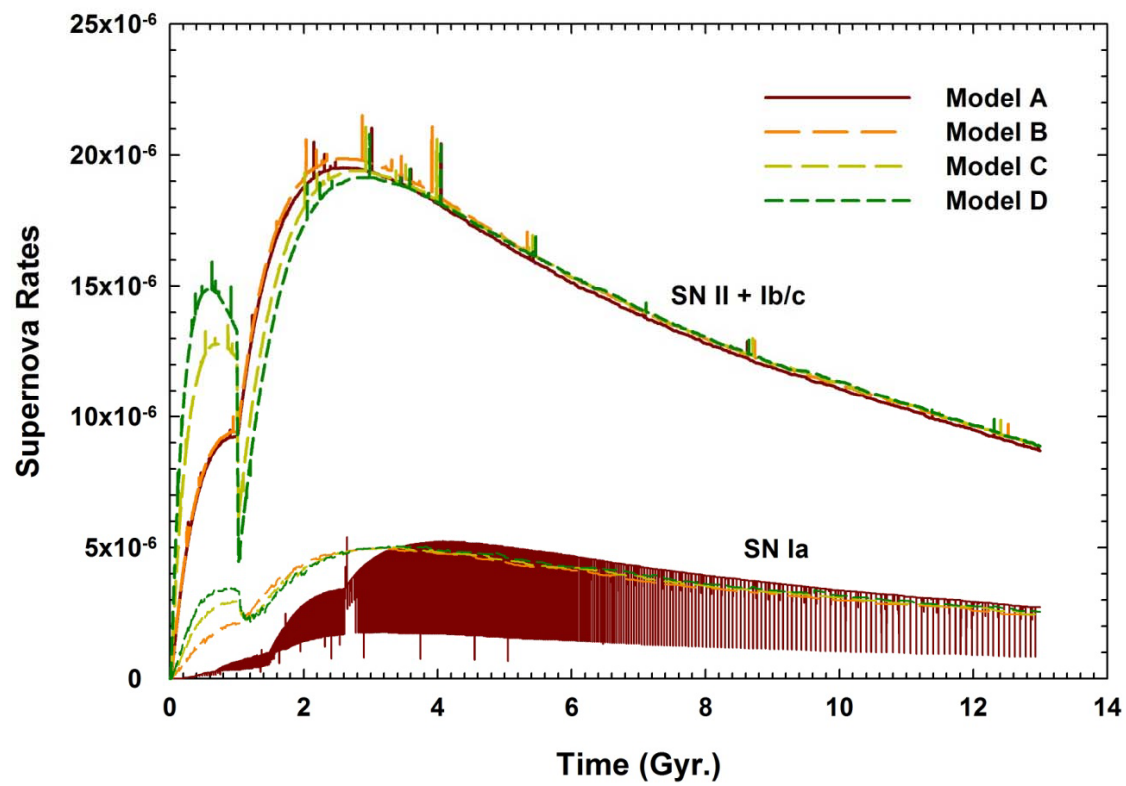

Figure 5. The deduced supernovae rates $\left(\mathrm{pc}^{-2} \mathrm{Myr}^{-1}\right)$ for the various models in the solar annular ring. The present predicted rates are in accordance with the observations [20].

the observed data of the elemental abundance distribution of dwarf stars in the solar neighborhood is also presented in Figure 6. Based on the comparison among Models A and B (Figure 6), due to the increase in the early enhanced production of iron during the initial 1 Gyr. (Figure 4) by prompt SN Ia (Figure 5) the mismatch between the predicted and observed elemental evolutionary trends become large in the case of Model B except for nitrogen and nickel. The elemental evolutionary trends in nickel are almost identical in the three models B-D with prompt SN Ia. Compared to Model B, Models C and D with the enhanced star formation rate in the initial $1 \mathrm{Gyr}$. (Figure 1), and hence an early enhanced SN II + I b/c rates, yield better match between the predicted and the observed elemental evolutionary trends except for nitrogen. 


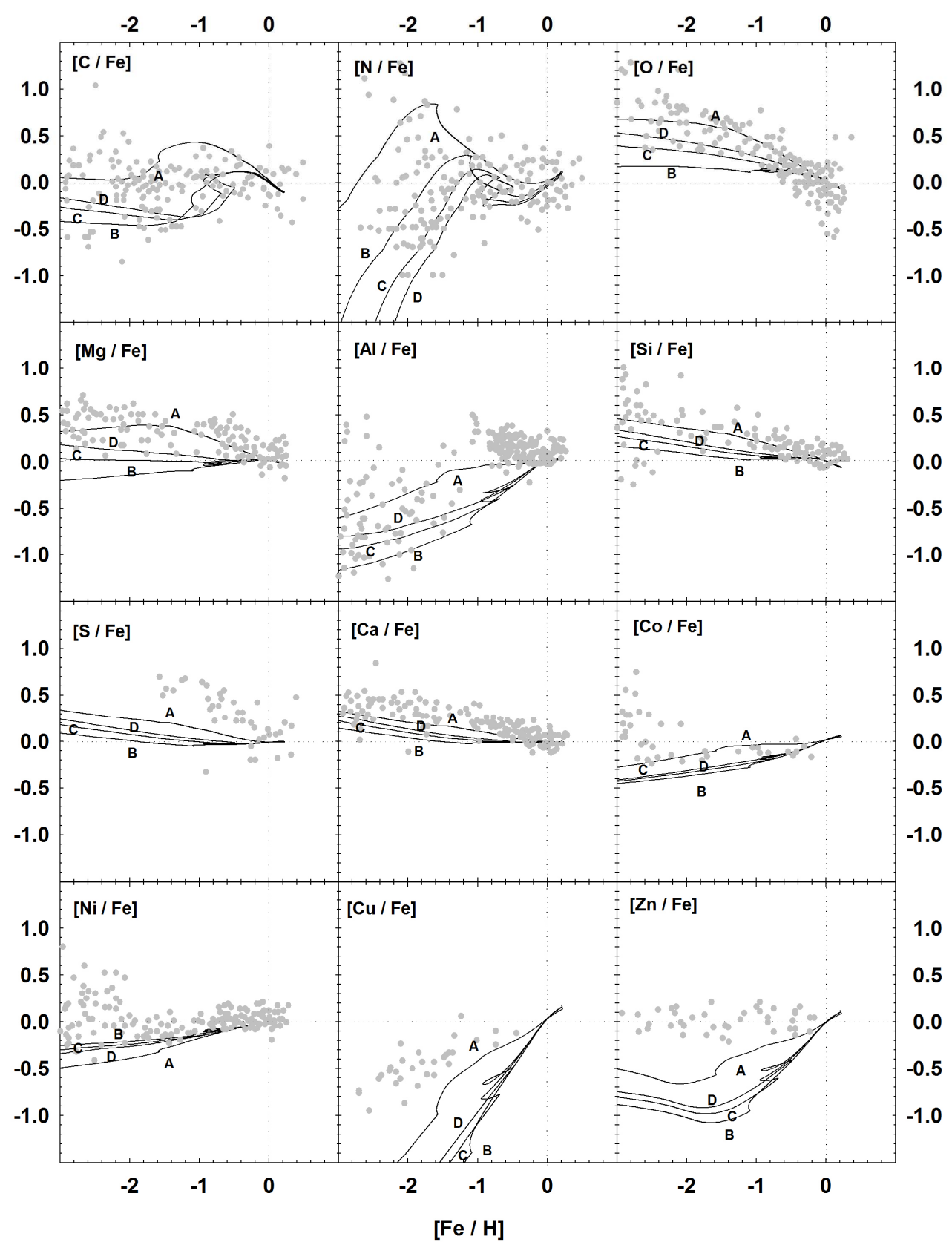

Figure 6. The deduced normalized elemental evolution of the various elements. The observed elemental abundance distributions of the dwarf stars are also presented for comparison (see [9] for detailed references).

The most significant among all the elements considered in GCE is oxygen that is mostly produced by $\mathrm{SN}$ II + $\mathrm{Ib} / \mathrm{c}$, whereas, iron is mostly produced by SN Ia. The relative production of oxygen and iron is critically important to understand the GCE (Figure 6). It should be noted that Model A (Table 1) makes the best prediction for the observed $[\mathrm{O} / \mathrm{Fe}]$ trend. This is followed by Model $\mathrm{D}$ with an enhanced star formation rate during the initial 1 Gyr.

On the basis of the $[\mathrm{Fe} / \mathrm{H}]$ evolutionary trends (Figure 4) and the elemental abundance evolutions (Figure 6), specifically the evolution of $[\mathrm{O} / \mathrm{Fe}]$, it appears that the SN Ia formulations with the prompt + delayed contributions of SN Ia would be able to explain most of the observational features of the dwarf stars in the solar neighborhood provided we also invoke an enhanced star formation rate at least by a factor of 3 as in the case of Model D during the earliest epoch of the galaxy.

Finally, it should be mentioned that the SN Ia formulation adopted in the previous study [9] could explain the observed elemental abundance distribution for most of the elements (Figure 6) except for a low initial iron abun- 
dance evolution. In fact, Model D with the best GCE predictions in the present work closely resemble with Model A that is based on the SN Ia formulation adopted earlier (Figure 6).

\section{Summary}

Based on a recently developed alternative approach for understanding the galactic chemical evolution in the solar neighborhood we examine the dependence of the chemical evolution on the SN Ia rates and the star formation rate during the earliest stages of the galaxy. The analysis favors the incorporation of the prompt SN Ia models that can occur within a time span of $\sim 100$ Myr. from the star formation. Further, the higher star formation rate during the initial one billion years, the epoch corresponding to the formation of the galactic halo and the thick disk, would be able to produce most of the elemental evolutionary trends except for few exceptions. The enhancement trend in the star formation rate should be as much as three times the trend prevailing in the latter time of the galaxy evolution.

\section{Acknowledgements}

This works was supported by the Planetary Sciences and Exploration (PLANEX) research grant of the Indian Space Research Organization (ISRO).

\section{REFERENCES}

[1] B. E. J. Pagel, "Nucleosynthesis and the Chemical Evolution of Galaxies," Cambridge University Press, Cambridge, 1997.

[2] F. Matteucci, "The Chemical Evolution of the Galaxy," Kluwer Academic Publishers, Dordrecht, 2003. doi:10.1007/978-94-010-0967-6

[3] F. X. Timmes, S. E. Woosley and T. A. Weaver, "Galactic Chemical Evolution: Hydrogen through Zinc," The Astrophysical Journal Supplement, Vol. 98, No. 2, 1995, pp. 617-658. doi:10.1086/192172

[4] C. Chiappini, F. Matteucci and R. Gratton, "The Chemical Evolution of the Galaxy: The Two-Infall Model," The Astrophysical Journal, Vol. 477, No. 2, 1997, pp. 765780. doi: $10.1086 / 303726$

[5] A. Alibés, J. Labay and R. Canal, "Galactic Chemical Abundance Evolution in the Solar Neighborhood up to the Iron Peak," Astronomy and Astrophysics, Vol. 370, No. 3, 2001, pp. 1103-1121 doi:10.1051/0004-6361:20010296

[6] F. Matteucci, E. Spitoni, S. Recchi and R. Valiante, "The Effect of Different Type Ia Supernova Progenitors on Galactic Chemical Evolution," Astronomy and Astrophysics, Vol. 501, No. 2, 2009, pp. 531-538. doi:10.1051/0004-6361/200911869

[7] C. Kobayashi and K. Nomoto, "The Role of Type Ia Supernovae in Chemical Evolution. I. Lifetime of Type Ia Supernovae and Metallicity Effect," The Astrophysical
Journal, Vol. 707, No. 2, 2009, pp. 1466-1484. doi:10.1088/0004-637X/707/2/1466

[8] C. Kobayashi and N. Nakasato, "Chemodynamical Simulations of the Milky Way Galaxy," The Astrophysical Journal, Vol. 729, No. 1, 2011, pp. 16-32. doi:10.1088/0004-637X/729/1/16

[9] S. Sahijpal and G. Gupta, "Numerical Simulation of the Galactic Chemical Evolution: The Revised Solar Abundance," Meteoritics and Planetary Science Journal, Vol. 48, No. 6, 2013, pp. 1007-1033. doi:10.1111/maps.12123

[10] M. Asplund, N. Grevesse, A. J. Sauval and P. Scott, "The Chemical Composition of the Sun," Annual Review of Astronomy and Astrophysics, Vol. 47, No. 1, 2009, pp. 481522. doi:10.1146/annurev.astro.46.060407.145222

[11] E. Anders and N. Grevesse, "Abundances of the Elements -Meteoritic and Solar," Geochimica et Cosmochimica Acta, Vol. 53, No. 1, 1989, pp. 197-214. doi:10.1016/0016-7037(89)90286-X

[12] B. Edvardsson, J. Andersen, B. Gustafsson, D. L. Lambert, P. E. Nissen and J. Tomkin, "The Chemical Evolution of the Galactic Disk-Part One-Analysis and Results," The Astrophysical Journal, Vol. 275, No. 1, 1993, pp. 101-152.

[13] H. Meusinger, B. Stecklum and H. G. Reimann, "The AgeMetallicity-Velocity Dispersion Relation in the Solar Neighborhood and a Simple Evolution Model," Astronomy and Astrophysics, Vol. 245, No. 1, 1991, pp. 57-74.

[14] H. J. Rocha-Pinto, W. J. Maciel, J. Scalo and C. Flynn, "Chemical Enrichment and Star Formation in the Milky Way Disk. I. Sample Description and Chromospheric AgeMetallicity Relation," Astronomy and Astrophysics, Vol. 358, No. 3, 2000, pp. 850-868.

[15] S. Sahijpal, "Galactic Chemical Evolution: The Star Formation Rate in the Early Galaxy," 39th COSPAR Scientific Assembly, Mysore, 14-22 July 2012, p.1651.

[16] M. A. Dopita and S. D. Ryder, "On the Law of Star Formation in Disk Galaxies," The Astrophysical Journal, Vol. 430, No. 1, 1994, pp. 163-178. doi:10.1086/174390

[17] A. I. Karakas and J. C. Lattanzio, "Stellar Models and Yields of Asymptotic Giant Branch Stars," Publications of the Astronomical Society of Australia, Vol. 24, No. 3, 2007, pp. 103-117. doi:10.1071/AS07021

[18] S. E. Woosley and T. A. Weaver, "The Evolution and Explosion of Massive Stars. II. Explosive Hydrodynamics and Nucleosynthesis," The Astrophysical Journal Supplement, Vol. 101, 1995, pp. 181-235. doi:10.1086/192237

[19] K. Iwamoto, F. Brachwitz, K. Nomoto, N. Kishimoto, H. Umeda, W. R. Hix and F. K. Thielemann, "Nucleosynthesis in Chandrasekhar Mass Models for Type Ia Supernovae and Constraints on Progenitor Systems and Burning-Front Propagation," The Astrophysical Journal Supplement, Vol. 125, No. 2, 1999, pp. 439-462. doi: $10.1086 / 313278$

[20] G. A. Tammann, W. Loefler and A. Schroder, "The Galactic Supernova Rate," The Astrophysical Journal Supplement, Vol. 92, No. 2, 1994, pp. 487-493. doi:10.1086/192002 